\title{
The Implication of Gastric Dysmotility in the Pathophysiology of Functional Dyspepsia
}

\author{
Yang Won Min \\ Department of Medicine, Samsung Medical Center, Sungkyunkwan University School of Medicine, Seoul, Korea
}

\section{Article: Prevalence of gastric motility disorders in patients with functional dyspepsia \\ Asano $\mathrm{H}$, Tomita T, Nakamura $\mathrm{K}$, et al \\ (J Neurogastroenterol Motil 2017;23:392-399)}

As functional dyspepsia (FD) is defined on the basis of symptom aggregations, it encompasses a number of pathophysiological causes. ${ }^{1}$ Conventionally, gastric dysmotility including delayed emptying and impaired accommodation has been proposed as the major mechanism to underlie symptom generation in FD. Thus, several prokinetics and fundus-relaxing drugs have been introduced but most of them show disappointing therapeutic effects and are not widely available due to adverse effects. ${ }^{2,3}$

FD contains heterogeneous patient populations. This disease entity is bad for drugs that had a single primary effect (acidsuppressive, prokinetic, fundus-relaxing, and psychotropic drugs). Nevertheless, acotiamide showed a therapeutic benefit in patients with FD. ${ }^{4}$ Patients receiving acotiamide for 4 weeks showed positive overall treatment efficacy in $52.2 \%$, which was $17.4 \%$ better than placebo. Acotiamide enhances gastric emptying and accommodation in patients with $\mathrm{FD} .^{5}$ Its multiple action mechanisms may contribute to the apparent clinical benefit for patients with FD. ${ }^{4}$ The study was conducted in a subgroup of FD patients who had meal-induced symptoms in whom a homogenous underlying pathophysiology was expected. ${ }^{4}$ However, the absolute benefit over placebo was modest. If it is true that enhanced motor functions were reflected in symptom improvement, therapeutic effects could get better when segregating patients with gastric dysmotility. Symptoms of FD could not predict the underlying pathophysiology. ${ }^{6}$ In a recent large study to investigate the gastric sensorimotor dysfunction in patients with $\mathrm{FD}$, the prevalence of impaired gastric accommodation and delayed emptying does not differ between postprandial distress syndrome and epigastric pain syndrome groups according to Rome III criteria. ${ }^{7}$

Delayed gastric emptying and impaired gastric accommodation are found in about $40 \%$ of patients with FD. However, the prevalence of gastric dysmotility may be different among the populations. Asano et $\mathrm{al}^{8}$ published in this edition of the Journal of Neurogastroenterology and Motility their own study that investigated the association between the prevalence of gastric motility abnormalities and gastrointestinal symptoms. Gastric emptying and accommodation were assessed by scintigraphic imaging and impaired in $14.9 \%$ and $10.6 \%$ of the study population, respectively. However, they could find no association between gastric motility abnormalities and symptoms. The authors should be congratulated on their meaningful work. Recognizing the prevalence of gastric dysmotility could make an optimal treatment strategy in patients with FD. According

Received: June 9, 2017 Revised: June 11, 2017 Accepted: June 13, 2017

@) This is an Open Access article distributed under the terms of the Creative Commons Attribution Non-Commercial License (http://creativecommons. org/licenses/by-nc/4.0) which permits unrestricted non-commercial use, distribution, and reproduction in any medium, provided the original work is properly cited.

*Correspondence: Yang Won Min, MD, PhD

Department of Medicine, Samsung Medical Center, Sungkyunkwan University School of Medicine, 81 Irwon-ro, Gangnam-gu, Seoul 06351, Korea

Tel: +82-2-3410-3409, Fax: +82-2-3410-6983, E-mail: yangwonee@gmail.com 
to the results, treatment of FD using prokinetic drugs would be disappointing in Japan. However, there are a few things to consider in the study by Asano et al. ${ }^{8}$ Test methods has a crucial impact on the results. The 2-hour gastric emptying time measurement may contribute to the relatively low prevalence of delayed gastric emptying, and scintigraphy was not optimal for evaluating gastric accommodation. In addition, a single center study design may have resulted in selection bias as addressed by authors.

Treatment of FD is a frustrating task due to its multifactorial nature. Specific pathophysiology of symptom generation including gastric motility abnormalities should be a pharmacologic target to satisfy patients with FD. Thus, we should continue to try subgrouping patients with $\mathrm{FD}$ according to the underlying pathophysiology. In the meantime, effective drugs that are highly selective for therapeutic target need to be developed to shed light on the treatment of FD.

Financial support: None.

\section{Conflicts of interest: None.}

\section{References}

1. Talley NJ, Ford AC. Functional dyspepsia. N Engl J Med 2015; 373:1853-1863.

2. Talley NJ, Tack J, Ptak T, Gupta R, Giguère M. Itopride in functional dyspepsia: results of two phase III multicentre, randomised, doubleblind, placebo-controlled trials. Gut 2008;57:740-746.

3. Vakil N, Laine L, Talley NJ, et al. Tegaserod treatment for dysmotilitylike functional dyspepsia: results of two randomized, controlled trials. Am J Gastroenterol 2008;103:1906-1919.

4. Matsueda K, Hongo M, Tack J, Saito Y, Kato H. A placebo-controlled trial of acotiamide for meal-related symptoms of functional dyspepsia. Gut 2012;61:821-828.

5. Kusunoki H, Haruma K, Manabe N, et al. Therapeutic efficacy of acotiamide in patients with functional dyspepsia based on enhanced postprandial gastric accommodation and emptying: randomized controlled study evaluation by real-time ultrasonography. Neurogastroenterol Motil 2012;24:540-545, e250-e251.

6. Karamanolis G, Caenepeel P, Arts J, Tack J. Association of the predominant symptom with clinical characteristics and pathophysiological mechanisms in functional dyspepsia. Gastroenterology 2006;130:296-303.

7. Vanheel H, Carbone F, Valvekens L, et al. Pathophysiological abnormalities in functional dyspepsia subgroups according to the Rome III criteria. Am J Gastroenterol 2017;112:132-140.

8. Asano H, Tomita T, Nakamura K, et al. Prevalence of gastric motility disorders in patients with functional dyspepsia. J Neurogastroenterol Motil 2017;23:392-399. 